\title{
Voluntariado em Saúde na Graduação e a Formação de Valores Humanísticos, Sigilo, Privacidade e Confidencialidade de Pacientes
}

\author{
Siomara Roberta de Siqueira ${ }^{1^{*}}$, Elma Lourdes Campos Pavone Zoboli², Silvia Helena Bastos \\ de Paula ${ }^{3}$ \\ ${ }^{1}$ Instituto Adolfo Lutz \\ ${ }^{2}$ Escola de Enfermagem da Universidade de São Paulo \\ ${ }^{3}$ Instituto de Saúde SES-SP
}

* Autora para correspondência: siqueirasiomara@gmail.com

\begin{abstract}
RESUMO
Voluntário é um termo polissêmico, mas, de forma geral, designa o cidadão que doa tempo, trabalho e talento, espontaneamente e sem remuneração, para causas de interesse comum. Este artigo tem sua base em estudo de tese sobre os riscos à privacidade, sigilo e confidencialidade de pacientes atendidos por voluntários na área da saúde, cujo recorte aqui apresentado trata do potencial dessa experiência de pesquisa e do campo na formação de profissionais de saúde. Os princípios da confidencialidade são de universal importância para a enfermagem, médicos, profissionais de saúde e voluntários. Em linhas gerais, trata-se de estudo de caso descritivo-analítico, com base em método misto, feito por meio de entrevista semiestruturada e aplicação dos instrumentos, adaptados e validados para a população brasileira: Critério de Classificação Econômica Brasil; Inventário de Funções do Voluntariado; e Questionário de Perfis de Valores Refinado. Foram realizadas a análise de conteúdo dos discursos e a análise estatística dos questionários. Há poucos trabalhos publicados no sentido de conhecimento sobre as práticas e sobre a divulgação dos aspectos jurídicos envolvidos no trabalho voluntário, com lacuna na divulgação das legislações existentes que possam sinalizar os passos para os quais os gestores de saúde devem atentar para dar apoio ao trabalho voluntário na saúde. O voluntariado se constitui em oportunidade de o aluno de graduação vivenciar a realidade de uma instituição e desenvolver na sua formação os valores éticos e humanísticos de compromisso com o outro como exercício de cidadania, para além da relação profissional.
\end{abstract}

Palavras-Chave: Enfermagem em Saúde Comunitária; Valores Sociais; Trabalhadores Voluntários de Hospital; Motivação; QPV-RR.

\begin{abstract}
Voluntary is a polysemic term, but, in general, it designates the citizen who donates time, work and talent, spontaneously and without remuneration, for causes of common interest. This article is based on a thesis on the risks to privacy, secrecy and confidentiality of patients treated by volunteers in the health area, whose presentation here addresses the potential of this research experience and the field in the training of health professionals. The principles of confidentiality are of universal importance to nursing, doctors, health professionals and volunteers. Descriptive-analytical case study, based on Mixed Method, made through semi-structured interview and application of the instruments, adapted and validated for the Brazilian population: Criterion of Economic Classification Brazil; Inventory of Volunteer Functions and Refined Values Profiles Questionnaire. The analysis of the content of the speeches and statistical analysis of the questionnaires was done. There are few published works in the sense of knowledge about practices and the dissemination of legal aspects involved in voluntary work, with a gap in the dissemination of existing legislation that can signal the steps that health managers should consider to support voluntary work in health. Volunteering is an opportunity for undergraduates to experience the reality of an institution and to develop in their formation the ethical and humanistic values of commitment to the other as an exercise of citizenship, in addition to the professional relationship.
\end{abstract}

Keywords: Community Health Nursing; Social Values; Hospital Volunteers; Motivation. 


\section{Introdução}

Este trabalho teve origem no projeto de tese de doutorado sob o tema "Motivação para o Trabalho dos Voluntários que Atuam em Hospital Público Estadual de São Paulo, Referência em HIV", com a orientação da Dra. Elma Lourdes Campos Pavone Zoboli, na linha de cuidados em Saúde, dentro do Programa de Pós-Graduação em Enfermagem - Interunidades.

Na construção da tese, deparamos com aspectos éticos enfrentados pelos voluntários que atuam na área da saúde. Os principais aspectos tratados no estudo foram: o trabalho voluntário, seus aspectos históricos, a motivação para o trabalho voluntário, aspectos de sua organização, o trabalho voluntário no Brasil, o trabalho voluntário na área da saúde e o voluntariado na Aids.

Neste artigo, discutem-se os princípios da privacidade e da confidencialidade no âmbito das relações entre usuários, profissionais e instituições de saúde, analisando seus aspectos conceituais e possibilidades de aplicação prática em atividades complementares de formação.

As repercussões do movimento em prol da atuação voluntária podem ser reconhecidas com a proclamação da Organização das Nações Unidas (ONU), que estabeleceu 2001 como Ano Internacional do Voluntariado, quando declarou 5 de dezembro como "Dia Internacional do Voluntariado" (ONU, 2013). Existem na graduação oportunidades de os discentes atuarem como voluntários em diversos países do mundo, com destaque para instituições americanas com longa tradição nesse tipo de atividade cidadã. Em São Paulo, existem projetos vinculados a cursos de graduação na área da saúde que criam e estimulam a participação social de estudantes, que representam 11,57\% do volume de pessoas envolvidas com voluntariado, e entre os profissionais 3,17\% são estudantes de enfermagem (MARQUES, 2006).

Voluntariado é uma palavra polissêmica. Significa um conjunto de ações desenvolvido pelo indivíduo voluntário, sem qualquer espécie de retribuição remunerada, financeira, recompensa material ou contrapartida, pressupondo uma decisão consciente, deliberada e livre do indivíduo (Idem, ibidem).
A Organização Mundial de Saúde (OMS) considera o voluntariado como elemento importante para a manutenção do bem-estar e da qualidade de vida na velhice, sendo uma proposta para o envelhecimento ativo. $\mathrm{O}$ voluntariado seria um processo de otimização das oportunidades de saúde, participação e segurança, cujo objetivo é manter a qualidade de vida à medida que as pessoas envelhecem (OMS, 2005). A palavra ativo refere-se à participação contínua nas questões sociais, econômicas, culturais, espirituais, civis, e não somente ao fato de estar fisicamente ativo ou de participar da força de trabalho (CALDANA \& FIGUEIREDO, 2008).

$\mathrm{O}$ objetivo geral da referida tese foi analisar as motivações para o voluntariado em um hospital público da cidade de São Paulo, especializado em HIV/ Aids. Teve também como objetivos específicos:

- Descrever características socioeconômicas e demográficas do voluntariado em um hospital público da cidade de São Paulo, especializado em HIV/Aids;

- Reconhecer a visão de tais voluntários acerca do trabalho voluntário;

- Identificar as funções do voluntariado nesse hospital público, especializado em HIV/Aids.

- Identificar valores motivacionais do voluntariado no referido hospital.

A pesquisa foi realizada em um hospital na cidade de São Paulo, referência em HIV, e o período de coleta de dados se estendeu de 23 de fevereiro a 19 de junho de 2015, com a participação de 160 voluntários.

Tendo em vista o objetivo da revista Grad+, este artigo propõe a apresentação de técnicas utilizadas na tese que, eventualmente, podem servir de modelo ou parâmetro para outros docentes, departamentos e instituições. Ao se estudar o objeto voluntariado, pretende-se contribuir para os estudantes de graduação, uma vez que o envolvimento com trabalho voluntário se apresenta como uma oportunidade de vivenciar a realidade do universo em que vão atuar após a conclusão do curso e como um desafio para esses jovens que, assim estimulados, podem compor propostas de intervenções específicas que respondam à necessidade social das comunidades. 


\section{Desenvolvimento}

Os instrumentos para coleta de dados da pesquisa foram: entrevista individual semiestruturada; questionário para caracterização do perfil socio demográfico do voluntário com base no Critério de Classificação da Associação Brasileira de Empresas de Pesquisa (Abep); Inventário de Funções do Voluntariado (IFV) adaptado para o Brasil (PILATI \& HESS, 2011); e Questionário de Perfis de Valores Refinado (QPV-RR), validado para o Brasil (TORRES, SCHWARTZ \& NASGIMENTO, 2016). Esses instrumentos foram apresentados nos Anexos da tese.

Os diferentes instrumentos usados na coleta de dados tinham o propósito de viabilizar informações para as análises quantitativas e qualitativas por meio da triangulação de técnicas (Quadro 1).

Para a caracterização socioeconômica, usou-se a classificação econômica da Associação Brasileira de Empresas de Pesquisa (ABEP, 2015). Essa classificação baseia-se nos critérios de consumo de produtos e serviços, tomando como unidade de análise o domicílio. Com isso, consegue-se a identificação de grandes grupos populacionais diferentes entre si.

O IFV e o QPV-RR são instrumentos para exploração dos valores e motivações, como descritos no apartado do marco teórico da tese. Todos os participantes responderam aos instrumentos e foram entrevistados.

\begin{tabular}{|c|c|c|c|}
\hline Técnicas & Instrumentos & Propósitos & Análise dos dados \\
\hline $\begin{array}{c}\text { Entrevista } \\
\text { semiestruturada. }\end{array}$ & $\begin{array}{l}\text { Roteiro norteador } \\
\text { para entrevista } \\
\text { semiestruturada com } \\
\text { gravação. }\end{array}$ & $\begin{array}{c}\text { - Identificação } \\
\text { de características } \\
\text { motivacionais do perfil } \\
\text { do voluntariado; } \\
\text { - Métodos mistos. }\end{array}$ & $\begin{array}{l}\text { Análise qualitativa, } \\
\text { utilizando Análise de } \\
\text { Conteúdo Temática. }\end{array}$ \\
\hline $\begin{array}{c}\text { Aplicação do } \\
\text { Questionário Abep. }\end{array}$ & $\begin{array}{c}\text { Critério de classificação } \\
\text { da Associação Brasileira } \\
\text { de Empresas de Pesquisa } \\
\text { (Abep) - LSE } 2015 .\end{array}$ & $\begin{array}{l}\text { - Identificação } \\
\text { de características } \\
\text { socioeconômicas } \\
\text { e demográficas do } \\
\text { voluntariado; } \\
\text { - Métodos mistos. }\end{array}$ & $\begin{array}{l}\text { Análise quantitativa, } \\
\text { utilizando SPSS. }\end{array}$ \\
\hline Aplicação do IFV. & $\begin{array}{l}\text { Inventário de Funções } \\
\text { do Voluntariado (IFV). }\end{array}$ & $\begin{array}{c}\text { - Identificação das } \\
\text { Funções do Voluntariado } \\
\text { para a população } \\
\text { estudada. }\end{array}$ & $\begin{array}{l}\text { Análise quantitativa, } \\
\text { estatística, a fim de } \\
\text { obter: Informações para } \\
\text { a criação dos escores } \\
\text { fatoriais; } \\
\text { - Correção da escala } \\
\text { segundo Pilati e Hess. }\end{array}$ \\
\hline Aplicação do QPV-RR. & $\begin{array}{l}\text { Questionário de Perfis } \\
\text { de Valores (QPV-RR). }\end{array}$ & $\begin{array}{l}\text { - Identificação dos } \\
\text { valores motivacionais } \\
\text { para o voluntariado na } \\
\text { população estudada. }\end{array}$ & $\begin{array}{l}\text { Análise quantitativa, } \\
\text { estatística, a fim de } \\
\text { obter a correção do } \\
\text { questionário. }\end{array}$ \\
\hline
\end{tabular}

Quadro 1 - Técnicas e instrumentos de coleta de dados e técnicas de análise de dados a serem utilizados nesta pesquisa para o voluntariado. Fonte: SIQUEIRA, 2016. 
Os discursos dos participantes foram trabalhados pela análise de conteúdo segundo Bardin (1977). A análise de conteúdo temática seguiu a orientação de quantificar as coocorrências (aparição de duas ou várias unidades de registro na mesma unidade de contingência) para agrupá-las nas categorias e contabilizá-las. A frequência dos temas no discurso foi tomada como indicativo da importância destes na motivação para o grupo no trabalho voluntário.

A análise estatística dos resultados do IFV, dos dados sociodemográficos e QPV-RR seguiu as orientações dos propositores dos instrumentos, considerando as adaptações para a população brasileira (PILATI \& HESS, 2011; ABEP, 2015; EUROPEAN, 2016; TORRES, SCHWARTZ \& NASGIMENTO, 2016). Utilizou-se o SPSS (Statistical Package for the Social Sciences), um software aplicativo do tipo científico (PESTANA \& GAGEIRO, 2014). Essa análise teve colaboração do estatístico Bernardo dos Santos (EEUSP).

\section{Aspectos Éticos}

O projeto foi encaminhado para a apreciação da Plataforma Brasil, segundo as normativas da Resolução $n^{\circ}$ 466/12, do Conselho Nacional de Saúde, recebendo parecer favorável n. 884.468, datado de 25 de novembro de 2014. Os instrumentos deste estudo foram previamente autorizados pelos autores e pelos responsáveis pela adaptação do instrumento para a população brasileira.

\section{Alguns Aspectos da Privacidade e o Volun- tariado}

A última década assistiu a um rápido aumento do interesse e da preocupação quanto à proteção da privacidade das informações pessoais. Alguns países dispõem de legislação relacionada à privacidade, outros não. A proteção da privacidade das informações pessoais é geralmente aceita como um direito civil fundamental (ALIU, 2014).

Além dos dados do paciente, o voluntariado, no contato direto com este, conhece uma série de atributos pessoais: orientação sexual, etnia, opiniões políticas e religiosas, traços de personalidade, inteligência, felicidade, uso de substâncias aditivas, situação matrimonial e/ou separação dos pais, a idade e a identidade de gênero (SIQUEIRA, 2016).

Entretanto, em nenhum lugar essa questão é trazida para o foco mais claro do que no contexto de sistemas de informação de saúde. Pacientes esperam que a informação que eles compartilham com o seu médico e o entorno hospitalar deva ser respeitada e mantida em sigilo. Os dados contidos em âmbito hospitalar podem ser potencialmente sensíveis. A equipe de saúde, os prestadores de serviços de cuidados, os serviços de assistência, ao terem acesso à informação, precisam manter sigilo compatível com a ética (MILNES \& KENDAL, 2012; HERTZMAN et al., 2013).

O trabalho apresentado no I Congresso de Graduação da USP fez um recorte do estudo, em que se buscou estabelecer as competências do voluntariado e identificar na literatura nacional e internacional aspectos direcionados à privacidade. As bases utilizadas foram: Biblioteca Virtual em Saúde (BVS), Web of Science, Scopus, tendo como descritores os termos privacidade (privacy), confidencilidade (confidentiality), voluntariado (volunteering) e saúde (health).

$\mathrm{Na}$ abertura da privacidade, é necessário que se estabeleça um acordo de confidencialidade, um pacto. A privacidade e a confidencialidade são princípios complexos que se correlacionam entre si. A privacidade consiste no conjunto de informações sobre uma pessoa, a qual pode decidir mantê-las sob seu exclusivo controle, ou comunicá-las, decidindo em que medida e a quem, quando, onde e em que condições o outro poderá acessar as informações. Já a confidencialidade se refere à garantia (no sentido de confiança) de que as informações confiadas não serão reveladas sem prévia autorização da pessoa em questão. Ou seja, a privacidade das informações é um direito dos usuários do serviço, ao mesmo tempo que a confidencialidade é um dever dos profissionais em relação às informações geradas e confiadas no relacionamento profissional-usuário (MASSAROLLO et al., 2006). 


\section{Resultados}

Estudantes de graduação também compõem o universo da pesquisa (4\%), sendo que relatam ser o voluntariado uma importante vivência para o desenvolvimento de sua futura profissão.

A análise dos artigos focou o princípio da privacidade e o da confidencialidade nos voluntários que atuam na área da saúde, evidenciando suas aplicações e mecanismos que os resguardam, assim como suas características, no contexto das relações entre profissionais de saúde e pacientes.

A história do voluntariado tem sua origem ligada à religiosidade dos indivíduos, ao civismo e a outros aspectos mais voltados para o crescimento individual e para uma melhor vida em sociedade. No Brasil, os antecedentes do trabalho voluntário também se dão em iniciativas de caráter religioso, legitimados pelo Estado (PILLOTI \& RIZZINI, 2011).

O Brasil não dispõe de proteção adequada para dados de natureza pessoal. Ainda que se considerem as proteções à intimidade e à privacidade estabelecidas pela Constituição Federal de 1988 (CF/1988), pelo Código Civil (CG), pela Lei de Acesso à Informação (Lei no 12.527/11), e o amparo aos dados relativos a processos de consumo (nos ditames trazidos pelo Gódigo de Defesa do Consumidor/CDC), estamos distantes de legislações do cenário mundial, tais como as adotadas pela Comunidade Europeia, Canadá, Argentina, México, Uruguai, Peru, Chile e Estados Unidos da América (LIMA \& MONTEIRO, 2013).

A atividade voluntária é uma das formas de expressão viva do sentimento de solidariedade humana traduzido em ação. Manifesta-se na doação de horas de vida em favor do próximo, requerendo talento e habilidade na busca constante de excelência.

Apesar de o voluntariado já estar presente há muito tempo nas unidades de saúde, ainda é pouco conhecido quanto às atividades desenvolvidas. Os princípios de confidencialidade são de suma importância. Regras, protocolos e diretrizes precisam ser formulados e, quiçá, órgãos reguladores, códigos de conduta dos voluntários. $\mathrm{O}$ sigilo deve ser colocado como item da maior importância, e especialmente na arena da prestação de cuidados de saúde.

O enfoque na privacidade e na confidencialidade de informações sobressai quanto à área da saúde, afinal a segurança não é uma questão simplesmente tecnológica: "[...] a segurança é um problema em termos de pessoas, não em termos de tecnologias" (NINIMÄK, SAVOLAINEN \& FORSSTRÖM, 1998).

Ao ingressar voluntariamente em um hospital, o paciente consente explícita e implicitamente o acesso a sua pessoa, ciente de que estará sujeito a certas perdas de privacidade, porém a sua decisão de entrar no hospital não significa que concedeu acesso irrestrito a sua pessoa. Interessante observar que, quando se concede acesso a outras pessoas, mesmo ciente da perda de privacidade, é possível manter algum controle sobre as informações geradas, pelo menos em contextos terapêuticos, de diagnóstico e em pesquisas. Pois somente será acusada de violar os direitos de confidencialidade aquela pessoa a quem se consentiu o acesso às informações.

Beauchamp e Childress (2002) referem:

[...] devemos ressaltar que diversas concepções da ética do caráter exibem um padrão de convergência similar e que os apelos aos princípios são muitas vezes intercalados com apelos às virtudes. Aristóteles e Hume, por exemplo, com frequência concordam ao recorrer às virtudes e em suas concepções sobre elas, e, embora os dois sejam fundamentalmente teóricos das virtudes, ambos reconhecem a importância dos princípios normativos gerais. Também Kant e Mill exibem uma profunda preocupação com aquilo que Mill chama de "o cultivo geral da nobreza de caráter". Quase todas as grandes teorias convergem para a conclusão de que o mais importante elemento da vida moral de uma pessoa é um caráter desenvolvido que proporcione a motivação e a força interiores para fazer o que é certo e bom (pp. 541-542).

O Departamento de Saúde e Serviços Humanos (Health and Human Service - HHS), agência de 
proteção da saúde americana, descreve críticas para as iniciativas de proteção e privacidade dos pacientes. Formularam um documento que é uma proposta para a política de privacidade, modelo a ser usado por fornecedores de registros pessoais em saúde. Outro documento é um "framework" de privacidade para nortear o desenvolvimento da rede nacional de informação em saúde.

A segurança e a confidencialidade devem ser protegidas de acordo com padrões nacionais, mas, ao mesmo tempo, uma mudança de paradigma (propriedade x custódia) é necessária para que as organizações de assistência médica criem uma cultura de custódia, e não da propriedade, dos dados do paciente. Essa mudança será conseguida através da criação de modelos de controle compartilhados entre profissionais de saúde, pacientes e do público em geral. Os pacientes devem ter a capacidade de controlar o fluxo e de influenciar no acesso aos seus dados clínicos (CONN, 2008).

Boyd et al. (2007) apresentam um mecanismo de "intermediação honesta" ("honest broker") para manter a privacidade de atendimento ao paciente e à pesquisa acadêmica em saúde, visando à integração para troca de informações e dos dados em saúde entre sistemas de forma segura e confiável.

A interligação dos dados em saúde, com dados do recenseamento populacional, objetiva resolver a falta da informação sobre etnia nos procedimentos de rotina em saúde, registrados nos Sistemas Informatizados em Saúde (SIS), beneficiando, assim, pesquisas que envolvam, principalmente, os grupos étnicos minoritários (Boyd, 2007). Segundo o autor, um estudo na Escócia, que interligou dados em saúde e a informação de etnia de 4,6 milhões de pessoas, revelou informações importantes sobre a incidência e sobrevida após infarto agudo do miocárdio entre os sul-asiáticos. A técnica de interligação de dados, desenvolvida com a preocupação de proteger o anonimato das pessoas envolvidas (criptografia e procedimentos organizacionais, cuidadosamente elaborados para o registro da vinculação), garantiu o cumprimento da legislação de proteção de dados, recebendo aprovação reguladora apropriada.

Há poucos trabalhos publicados no sentido de conhecimento e divulgação dos aspectos jurídicos envolvidos no trabalho voluntário, sendo de suma importância difundir as legislações existentes, sinalizar os passos para os quais os gestores de saúde devem atentar para o trabalho voluntário na saúde.

Alguns autores, como Ferrarezzi e Oliveirra (2010), colocam em debate a reflexão sobre processos participativos e a emergência de novos padrões de relação entre o Estado e a sociedade. Segundo Goulart (2010):

Como estratégia de atenção à saúde, na qualidade de política de Estado, é necessário que a humanização seja implementada como uma política transversal, que atualiza um conjunto de princípios e diretrizes por meio de ações e modos de agir nos diversos serviços, práticas de saúde e instâncias do sistema, caracterizando uma construção coletiva. A humanização como política transversal supõe, necessariamente, ultrapassar as fronteiras, muitas vezes rígidas, dos diferentes núcleos de saber/poder que se ocupam da produção da saúde. Entendemos, entretanto, que tal situação de transversalidade não deve significar um ficar fora, ou ao lado, do Sistema Único de Saúde - SUS.

Na Constituição de 1998, o artigo 14 garantiu a iniciativa popular como principiadora de processos legislativos. O artigo 29, sobre a organização das cidades, requereu a participação dos representantes de associações populares no processo de organização das cidades. Outros artigos demandaram a participação das associações civis na implementação das políticas de saúde e assistência social.

$\mathrm{Na}$ sequência da análise da interconexão das três áreas (privacidade, qualidade e responsabilidade civil), eis um resumo das considerações das leis existentes e das propostas para a privacidade das informações em saúde: 1) reconhecer que a informação em saúde é altamente sensível; 2) fornecer garantias de privacidade com base em práticas 
leais de informação; 3) habilitar os pacientes com a informação e com direitos de consentimento para a divulgação; 4) limitar a divulgação de dados em saúde em que faltar consentimento; 5) incorporar recursos de proteção de segurança; 6) criar uma autoridade nacional de proteção de dados; 7) garantir um nível mínimo nacional de proteção à privacidade (ROSÁRIO, 2010).

No caso brasileiro, uma herança comum do processo de democratização fomentou atores sociais democráticos, especialmente provenientes do movimento comunitário, a disputarem o significado do termo participação (SANTOS, 2002).

$\mathrm{O}$ respeito à privacidade é valor central da moral comum anglo-americana. $\mathrm{O}$ modelo da lei de privacidade na saúde estadunidense considera que as informações de saúde identificáveis são dignas de tutela jurídica. A "Lei Modelo" aplica-se a todas as "informações de saúde protegidas" por agências de saúde pública. Trata-se de informações, sejam orais, escritas, eletrônicas ou visuais, que se relacionam com o passado de um indivíduo, o presente ou o futuro estatuto de saúde física ou mental, condição, tratamento, serviço, compras de produtos ou prestação de cuidados. Essa definição ampla de informações de saúde protegidas reconhece que quaisquer dados identificáveis podem ser sensíveis e necessitam de proteção (LAWRENCE et al., 2001).

Importante veicular a Lei n 9.608, de fevereiro de 1998, cujos artigos dispõem sobre o serviço voluntário e sobre outras providências.

Art. 1: Considera-se serviço voluntário, para fins desta Lei, a atividade não remunerada, prestada por pessoa física a entidade pública de qualquer natureza ou instituição privada de fins não lucrativos, que tenha objetivos cívicos, culturais, educacionais, científicos, recreativos ou de assistência social, inclusive, mutualidade.

\section{Conclusão}

Inegavelmente, constitui um avanço o respaldo jurídico à prestação de serviço voluntário, regulamentando a prática do voluntariado e protegendo as entidades de reclamações na Justiça Trabalhista.
Por outro lado, ficou uma lacuna. Não foram criadas facilidades, nem incentivos ao cidadão para que ele tenha ainda mais motivos - além dos ligados à solidariedade, à nobreza da causa e à satisfação pessoal - para se dedicar à atividade do serviço voluntário.

Como se salientou, além de conhecer vários aspectos do paciente, o voluntariado, no contato direto com o paciente devido naturalmente à sua proximidade, terá acesso direto ou indireto a uma série de atributos pessoais, tais como orientação sexual, etnia, opiniões políticas e religiosas, traços de personalidade, inteligência, felicidade, uso de substâncias ilícitas, situação matrimonial e/ou separação dos pais, a idade e a identidade de gênero (FORREST, 2011).

Os princípios da confidencialidade são de universal importância para a enfermagem, médicos, profissionais de saúde e voluntários. Novos caminhos, protocolos e guias de pesquisa precisam ser formulados refletindo os códigos de condutas profissionais, visando a proteger o acesso aos dados do paciente por pessoas não autorizadas.

\section{Referências Bibliográficas}

ALIU, O.; CORLEW, S. D.; HEILES, M. E.; PANNUCGI, G. J. \& CHUNG, K. C. "Building Surgical Capacity in Low-Resource Countries. A Qualitative Analysis of Task Shifting from Surgeon Volunteers' Perspectives". Annals of Plastic Surgery, vol. 72, n. 1, pp. 108-12, 2014.

ASSOCIAÇÃO BRASILEIRA DE EMPRESAS DE PESQUISA (ABEP). Critério de Classificação da Abep -Base LSE 2015. São Paulo: Abep, 2015. Disponível em: $\langle$ http://www.abep.org/criterio-brasil $>$. Acessado em 10 fev. 2015.

BARDIN, L. Análise de Conteúdo. São Paulo: Martins Fontes, 1977.

BEAUCHAMP, T. L. \& GHILDRESS, J. F. Princípios de Ética Biomédica. 4 ed. São Paulo: Loyola, 2002.

BOYD, A. D.; HOSNER, G.; HUNSCHER, D. A.; ATHEY, B. D.; CLAUW, D. J. \& GREEN, L. A. "An 'Honest Broker' Mechanism to Maintain Privacy for Patient Care and Academic Medical Research". International Fournal of Medical Informatics. Ireland, vol. 76, n. 5-6, pp. 407-411, 2007.

BOYD, K. M. "Ethnicity and the Ethics of Data Linkage". BioMed Central (BMC) Public Health, England, 2007. 
CALDANA, A. C. F. \& FIGUEIREDO, M. A. C. "O Voluntariado em Questão: a Subjetividade Permitida". Psicol Cienc Prof Brasilia [Internet], vol. 28, n. 3, pp. 466-479, 2008. Disponível em: http://www.scielo.br/pdf/ pcp/v28n3/v28n3a03.pdf. Acessado em 21 jul. 2014.

CONN, J. "Critics Assail HHS Initiative. Framework Described as Lacking Privacy Protections". Modern Healthcare, USA, vol. 38, n. 51, pp. 14-15, 2008.

EUROPEAN Social Survey. "Computing Scores for the 10 Human Values". 2012. Avaliable from: https://www.europeansocialsurvey.org/docs/methodology/ESS1_human_values_scale.pdf. Acessado em 15 jan. 2016.

FERRAREZI, E. \& OLIVEIRA, M. S. de C. Conferências Nacionais de Saúde e Processos Participativos na Administraşão Pública Federal Brasileira. Brasília: Escola Nacional de Administração Pública, 2010. Disponível em: <http://repositorio.enap.gov.br/handle/1/332>. Acessado em 20 dez. 2017.

FORREST, L. "Improving Patient Privacy and Confidentiality in One Regional Emergency Department - a Quality Project by Pauline Calleja". Australasian Emergency Nursing Fournal, 2011.

GOULART, M. S. B. \& Duraes F. "A Reforma Psiquiátrica e os Hospitais Psiquiátricos: Histórias da Desinstitucionalização". Psicologia e Sociedade. 22 (1):11220, 2010.

HERTZMAN et al. "Privacy by Designat Population Data Base: a Case Study Scribing the Technical, Administrative, and Physical Controls for Privacy-Sensitive Secondary Use of Personal Information for Research in the Public Interest". I Am Med Assoc Informe, vol. 20, pp. 25-28, 2013.

LAWRENCE, O.; GOSTIN, L. O.; HODGE, J. G. Jr. \& VALDISERRI, R. O. "Informational Privacy and the Public's Health: the Model State Public Health Privacy Act". Am f Public Health. vol. 91, n. 9, pp. 13861392, 2001.

LIMA, C. C. \& MONTEIRO, R. L. "Panorama Brasileiro sobre a Proteção de Dados Pessoais: Discussão e Análise Comparada". AtoZ: Novas Práticas em Informação e Conhecimento, [S.1.], vol. 2, n. 1, pp. 60-76, 2013. Disponível em: doi:http://dx.doi.org/10.5380/ atoz.v2i1.41320.

MARQUES, V. L. Voluntariado: Motivos e Repercussões na Vida Social e Acadêmica dos Alunos de Graduação de Medicina, Voluntários em Programas na Área da Saúde. Dissertação de Mestrado, Escola Paulista de Medicina, Universidade Federal de São Paulo, 2006 [citado 2014 jan 14]. Disponível em: http://www.hsp.epm.br/centros/cedess/ producao/teses/tese_p_38.pdf.

MASSAROLLO, M. K. B. et al. "Autonomia, Privacidade e Confidencialidade". In: OGUISSO, T. \& ZOBOLI, E. L. C. P. (orgs.). Ética e Bioética: Desafios para a Enfermagem e a Saúde. São Paulo: Manolle, 2006, pp. 136-152.

MILNES, L. \& KENDAL, S. "Ethical Challenges of Conducting Health Research in UK School Setting". British fournal of Nursing, vol. 21, n. 5, pp. 294-298, 2012.

NINIMÄKI, J.; SAVOLAINEN, M. \& FORSSTRÖM,J.J. "Methodology for Security Development of an Electronic Prescription System". In: Proceedings of AMIA Symposium, Orlando, 1998, pp. 245-249.

NORMA ABNT NBR ISO/IEC 27001: 2006 [gestão de segurança da informação, capacita o Datasus de forma necessária, para a garantia dos aspectos básicos de confidencialidade].

ORGANIZAÇ̃̃O DAS NAÇÕES UnIDAS (ONU). "El Tema de la Celebración del Día Internacional de los Voluntarios de 2013 Es: Jóvenes del Mundo Activos". ONU, 2013. Disponível em: <http://www.onu. $\mathrm{cl} /$ onu/dia-internacional-de-los-voluntarios-2/>. Acessado em 20 dez. 2017.

ORGANIZAÇÃO MUNDIAL DA SAÚDE (OMS). Envelhecimento Ativo: Uma Política de Saúde. Brasília: Organização Pan-Americana da Saúde, 2005. Disponível em: <http://bvsms.saude.gov.br/bvs/publicacoes/envelhecimento_ativo.pdf $>$. Acessado em 20 dez. 2017.

PESTANA, M. H. \& GAGEIRO, J. N. Análise de Dados para Ciências Sociais: a Complementaridade do SPSS. 6 ed. Lisboa: Edições Sílabo, 2014. Disponível em: http://www.silabo.pt/Conteudos/7752_PDF.pdf.

PILATI, R. \& HESS, M. A. G. "Evidências de Validade de uma Versão Brasileira do Inventário de Funções do Voluntariado - IFV". Psico-USF [Internet], vol. 13, n. 3, pp. 275-284, 2011. Disponível em: http:// dx.doi.org/10.1590/S1413-82712011000300004. Acessado em 15 dez. 2012.

PILOTTI, F. J. \& RIZZINI, I. A Arte de Governar Crianças: A História das Políticas Sociais, da Legislação e da Assistência à Infância no Brasil. São Paulo: Cortez, 2011, pp. 30-97.

ROSÁRIO, M. S. A Segurança das Informações em Saúde sob a Responsabilidade do Datasus: uma Análise com Enfoque na Privacidade e na Confidencialidade. Dissertação, Escola Nacional de Saúde Pública Sergio Arouca, Rio de Janeiro, 2010.

SANTOS, J. A. F. Estrutura de Posições de Classe no Brasil: Mapeamento, Mudanças e Efeitos na Renda. Belo Horizonte: Editora UFMG, 2002.

SIQUEIRA, S. R. Motivação para o Trabalho dos Voluntários que Atuam em Hospital Público Estadual de São Paulo, Referencia em HIV. Tese, Escola de Enfermagem da Universidade de São Paulo, 2016.

. \& ZOBOLI, E. L. C. P. "Voluntários em Serviços de Saúde: Implicações para a Privacidade dos Pacientes". In: KEINERT et al. (orgs.). Proteção à Priva- 
cidade e Acesso às Informações em Saúde: Tecnologias, Direitos e Ética. São Paulo: Instituto de Saúde, 2015, pp. 421-440.

TORRES, C. V.; SCHWARTZ, S. H. \& NASGIMENTO, T. G. "A Teoria de Valores Refinada: Associações com Comportamento e Evidências de Vali- dade Discriminante e Preditiva". Revista de Psicologia da USP, São Paulo, vol. 27, n. 2, pp. 341-356, ago. 2016. Disponível em: $<$ https://www.revistas.usp.br/psicousp/ article/view/119783>. Acessado em 15 nov. 2017.

Publicado em 22/12/2017. 